\title{
Non-LTE line transfer with spatially correlated turbulence: Fluctuation against average emergent radiation field
}

\author{
M. L. Loucif ${ }^{1,2}$ and L. Ben Jaffel ${ }^{2}$ \\ 1 Observatoire d'Alger, CRAAG, BP 63, Bouzareah-Alger, Algeria \\ 2 Institut d'Astrophysique de Paris - CNRS, 98bis Bld. Arago, 75014 Paris, France \\ e-mail: loucif@iap.fr; bjaffel@iap.fr
}

Received 1 March 2000 / Accepted 3 April 2002

\begin{abstract}
The increasing interest in using turbulence to explain anomalies observed in stellar spectral lines is discussed. This turbulence occurs at a finite spatial scale, a key parameter. In our previous work on non-LTE radiation transfer in the presence of a turbulent velocity field (Loucif \& Magnan 1982), we calculated individual profiles representing intensity fluctuations around the averaged line shape for photon diffusion with complete redistribution in frequency $(\mathrm{CFR})$, a function well-adapted to media where collisional excitations dominate. We now generalize the problem by accounting for partial redistribution in frequency (PFR). To show the effect of the spatial correlation in the velocity field on the emergent line profiles as well as the importance of fluctuations in the medium's optical properties against the average, exhaustive calculations of emergent non-LTE line profiles are performed in the frame of a systematic comparison between the two processes of redistribution for the three following cases: static medium, averaged turbulent medium, and deviation from the average. In all cases, we confirm the differences and similarities in CFR and PFR descriptions previously derived for static media. These results seem reasonable since light scattering occurs at the atomic level-a scale well below the macroscopic size of the turbulence scale length. In addition, pronounced differences in the emergent radiation field are obtained between the average case and the so-called deviation from average for both frequency redistribution functions. These systematic differences rule out classical treatment of line formation in turbulent fields that assume averaged media to model observations obtained over an exposure time shorter than the turbulent field time scale $\left(t \sim l / V_{\mathrm{T}}\right)$. Beyond the different assumptions made to describe the non-LTE line formation in a turbulent medium, our model provides a simple and coherent description of realistic media applicable to different astrophysical observational backgrounds. For this purpose, a unified picture is proposed for the radiation field formation, invoking mainly the medium opacity, the turbulence correlation length, and the velocity. Observations of variable objects, or along extended lines of sights, allowing a high probability for such deviations to occur (e.g., quasars), are suitable for application of our model.
\end{abstract}

Key words. line: formation - line: profiles - radiative transfer - turbulence - ISM: abundances stars: emission line, Be

\section{Introduction}

The use of turbulence processes in the treatment of the formation of spectral lines has now been extended beyond the unique frame of stellar atmospheres, and a consensus is emerging that a full account should be taken of the spatial structure of the velocity field by introducing a finite spatial correlation length between the turbulent velocities of the medium (see Loucif \& Magnan 1982; Magnan 1985; Albrech \& Kegel 1987; Piehler \& Kegel 1995; Levshakov et al. 1999, 2001; Hegmann \& Kegel 2000; references to previous works may be found at the end of a contribution by Sedlmayr 1980). Because all the relevant quantities of the non-LTE medium are fluctuating, resolution of the stochastic radiation transfer equations is difficult, and interest has turned toward the determination of the average emerging intensity. In a previous study, a sophisticated model that includes turbulence was proposed in order to compute the deviation from the averaged value of the intensity in the particular case of complete redistribution of frequency (CFR; Loucif \& Magnan 1982). The resulting spectral line profiles showed that the velocity field is a very critical parameter for determining the line 

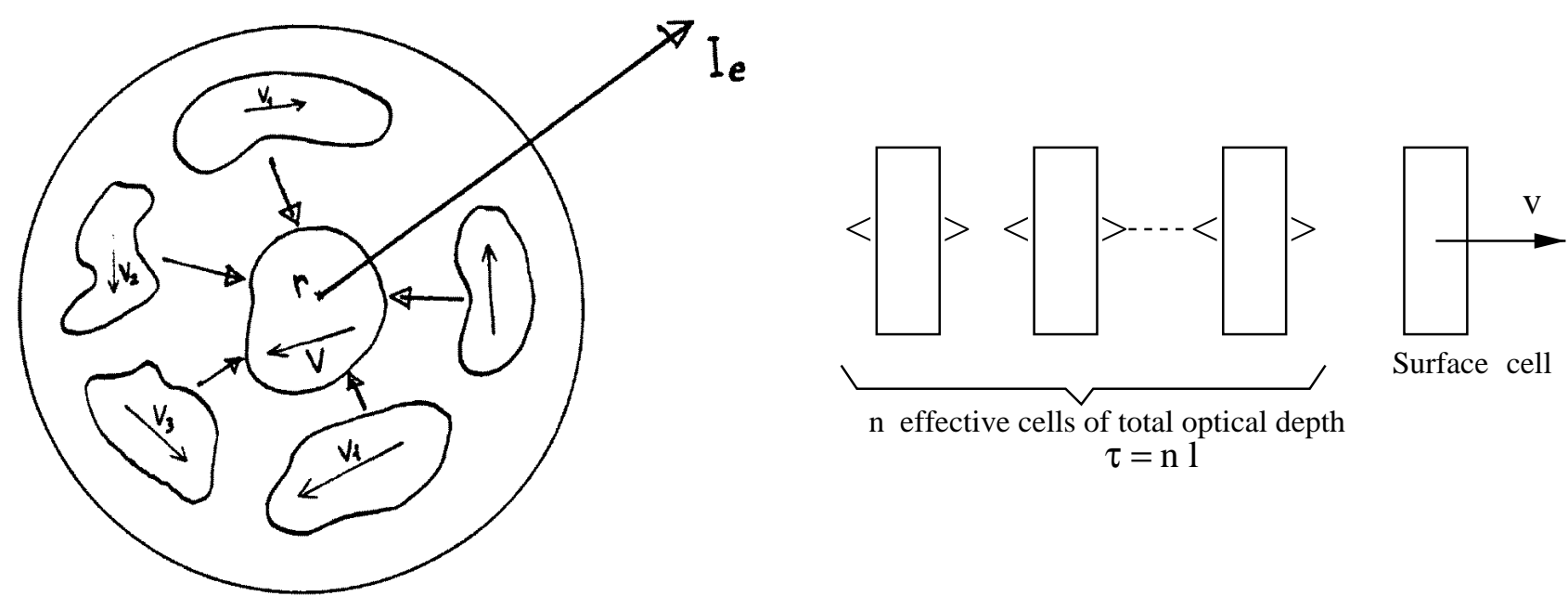

Fig. 1. Schematic representation of turbulent cells moving with particular velocities $v_{1}, \ldots, v_{n}$. Each cell receives energy from all the others, and the intensity $I e$ emerging from a given cell depends on all the cells' velocities in the medium (left). Schematic representation of the 1-D model (right).

shape, and compared well to emission lines observed from stars with high atmospheric velocities and (possibly) large mass ejection as well. In the solar example, the classical complexity of the highly resolved $\mathrm{CaII} \mathrm{H}$ and $\mathrm{K}$ chromospheric lines and other transition region lines (Vial et al. 1979), as well as the more recent extensive high-resolution observations of the Higher H Lyman series lines provided by the SUMER experiment on SOHO (see Ding et al. 1998; Gontikakis et al. 1997; Warren et al. 1998) indicate that such a radiative transfer model is needed (see also Mihalas 1978). Recent Hubble Space Telescope (HST) observations of Jupiter have also shown distorted Lyman- $\alpha$ emission line from the equatorial region of the planet with variations in the line shape on time scales of a few minutes (Emerich et al. 1996). Interestingly, the overall shape of the observed lines compares surprisingly well with the model calculations by Loucif \& Magnan of the deviation from the average line shape.

In this work, the problem is generalized to account for both partial and complete frequency redistribution of photons in the reference frame of the atoms applied to three specific cases: static medium, averaged turbulent medium, and deviation from the average. The last application is of special importance due to the progress in spatial/time resolution of modern observations (SOHO, HST, FUSE) that may help to discriminate between compelling linebroadening processes which may, in turn, greatly renew interest in studying fluctuating quantities against averaged ones. The sensitivity of the emergent radiation field to the medium opacity, turbulence velocity, and correlation length is shown. Key properties and behavior of the emergent intensity - of interest for observational purposes - are proposed.

\section{Description of the problem}

\subsection{Model's foundation}

The model, in its basic form, is well-described in Magnan (1976) where mean emergent profiles are determined for media with spatially correlated turbulence. The treatment of the deviation from the average is developed in Loucif \& Magnan (1982). We will describe below some essential aspects of the model for an understanding of the problem and then enumerate some approximations of a practical nature.

We consider an atmospheric medium in the presence of a completely non-thermal random velocity field. At different points of the medium, the velocities are not totally independent but have a certain spatial correlation. To take account for the spatial velocity correlation, the medium is divided into rigid volumes (or cells) of different sizes. In each point of a given volume, the velocity $v$ is constant, but it can take a completely different value on another volume of gas to remain constant there, and so on. The velocities of the different volumes are statistically independent and obey an assumed Gaussian distribution law characterized by the parameter $V_{\mathrm{T}}$, indicating the most probable velocity representing the velocity of turbulence:

$P(v) \mathrm{d} v=\left(V_{\mathrm{T}} \sqrt{\pi}\right)^{-1} \exp \left[-\left(v / V_{\mathrm{T}}\right)^{2}\right] \mathrm{d} v$.

We examine, then, the problem of transfer of radiation in a spectral line within a medium thus made up of turbulent cells. The specific question is: what radiation can be emitted by a particular cell having a particular velocity $\boldsymbol{v}$ (see Fig. 1)? The incident radiation $I_{\mathrm{i}}$ on a given cell depends on the velocity of all the other cells that contribute 
to this radiation (including the velocity of the particular cell considered - see Loucif \& Magnan 1982):

$I_{\mathrm{i}}=I_{\mathrm{i}}\left(v ; v_{1}, \ldots, v_{n}\right)$.

In the continuous exchange between radiation and matter, the velocity $v$ enters in the absorption coefficient which thus becomes a stochastic variable, and the intensity of the radiation that depends on it is then a fluctuating quantity. Traditionally, common practice has been to concentrate on the determination of the mean emergent intensity $\left\langle I_{\mathrm{e}}\right\rangle$, the quantity generally observed in stellar spectra (Albrech \& Kegel 1987; Levshakov et al. 2001). But in this work, we are instead interested in the deviation from this average: we calculate for given points (cells) of the medium individual spectral line profiles corresponding to a certain realization of the velocity field. This realization (an instantaneous photography of the turbulent medium) consists of the consideration of an ensemble of $n$ cells assigned of $n$ particular velocities $v_{1}, v_{2}, \ldots, v_{n}$ in which we determine the emergent intensity from a given cell (see Fig. 1). This determination of the emergent intensity $I_{\mathrm{e}}$, by taking account of all velocities of cells, is realizable in itself; however, in addition to its arbitrary character, it requires enormous calculations and is only of secondary interest. To solve the problem, we will easily show that by using the approximation of the effective cell (mean cell) described by Magnan (1976), the emergent radiation of a cell depends only on the velocity of the mean one, the other cells having been replaced by effective cells. One would then proceed as if the last cell, assigned a particular velocity $v$, were illuminated by an average radiation (Fig. 1).

\subsection{Physical processes}

We consider a typical simplified non-LTE case, where photons are created uniformly within the medium at the expense of thermal energy. In the layer of optical thickness $\tau$, a number of $\epsilon B \tau$ photons are emitted for the first time in each direction (the medium being considered as onedimensional). Afterward, the photons are allowed to diffuse in the medium, but at each scattering event there is a probability $\epsilon$ for the photon to be destroyed, its energy returning to the thermal pool. The probability of absorption within the medium is characterized by the shape factor $\phi_{x}=\int_{-\infty}^{+\infty} R\left(x, x^{\prime}\right) \mathrm{d} x^{\prime}$ at frequency $x$; the integration is done on the full emission frequencies. In the case of complete frequency redistribution, we have $R\left(x, x^{\prime}\right)=\phi_{x} \phi_{x^{\prime}}$, where $\phi_{x}$ is the Voigt function $U(b, x)$ and $b$ is the classical Voigt parameter. After absorption, the frequency distribution of the re-emitted photon is taken as the same function $U$ in the frame of reference of the matter at the point where the scattering occurs.

In the other considered case of the angles' averaged partial frequency redistribution (PFR), $R\left(x, x^{\prime}\right)=$ $R_{\mathrm{II}}\left(b, x, x^{\prime}\right)$ (Hummer 1962). The scattering is considered coherent in the atom's rest frame. In all cases, the frequencies are conveniently expressed in units of Doppler width as $x=v / a=(c / a)\left(\Delta \nu / \nu_{0}\right)$, where $a$ is the thermal velocity of the considered atom. The quantities $\epsilon$ and $b$ are both chosen as $10^{-3}$ in the results that follow.

\section{Method of solution and practical simplifications}

The problem of radiative transfer is examined in only one direction, and the variation of non-thermal velocities only considered according to this one direction of propagation of the light; we then calculate the radiation propagating through a column of cells that move randomly with respect to the line of sight. The dimension $l$ of a cell is constant, and we proceed on the given assumption that all the cells are identical. We use the method of addition of layers described by Sobolev (1963) in its simplest version: the doubling. The generic adding method applies to non-homogeneous media, but it is much more consumptive of CPU time. An essential parameter is the number $n$ of these cells, which can vary from $n=1$ (macroturbulence) to $n \rightarrow \infty$ (microturbulence). In a given realization, this number $n$ is such that $n l=\tau$, where $\tau$ is the total optical depth over which the radiation is integrated to the surface. The parameter $n$ thus makes it possible to explore all the realizations between the limits of micro- and macroturbulence (Fig. 1).

In this method of adding, suitable to our problem, we consider the emergent intensity $I_{\mathrm{e}}$ from a given cell as a function of the intensity $I_{\mathrm{i}}$ incident on that cell. In a concise way that can be applied to very general cases, we write for each cell a set of equations of the form

$$
I_{\mathrm{e}}(v)=E(v)+\{M(v)\} I_{\mathrm{I}}
$$

where $v$ is the velocity of the considered cell taken separately, $E$ is a constant term characterizing the emission of the cell when it is isolated, and $M$ is a matrix-operator which designs reflection and transmission matrices at the same time, connecting the outward intensity to the inward. Now, when the particular cell is moving in the turbulent medium (with the particular velocity $v$ ), the previous equation then becomes

$$
I_{\mathrm{e}}\left(v ; v_{1}, \ldots, v_{n}\right)=E(v)+\{M(v)\} I_{\mathrm{i}}\left(v ; v_{1}, \ldots, v_{n}\right)
$$

However, the difficulty of the non-LTE problem lies in the correlation between the intensity $I_{\mathrm{i}}$ incident on a cell and the operator $M$ characterizing the same cell. There exists a correlation because the intensity leaving the cell is likely to return onto the same cell it has left after scattering in the medium and will then keep a memory of the cell it has left, especially in regard to the velocity of the cell. The effective cell approximation introduced by Magnan (1976) consists precisely in neglecting the stated correlation. The essence of this approximation consists in supposing that if a given photon happens to come back to a cell it has crossed previously, then it sees a velocity that is statistically independent of the velocity which the cell had previously. Conversely, when a photon encounters a given velocity, it behaves as if it had never seen it before. 
By this approximation, the photon history is clearly reduced to a Markov process with no back-correlation. Thus, in particular, the intensity incident to a cell is independent of that cell's velocity: it remembers only the velocity of the cell it has immediately left (see also Loucif 1981, for more details). Using such an approximation, the equation finally becomes

$$
\begin{aligned}
<I_{\mathrm{e}}\left(v ; v_{1}, \ldots, v_{n}\right)>= & E(v)+\{M(v)\} \\
& <I_{\mathrm{i}}\left(v ; v_{1}, \ldots, v_{n}\right)>.
\end{aligned}
$$

As a consequence of the above approximation, the solution to the problem is obtained in the following way: the cell, at the surface where we want to determine the emerging intensity, is assigned a particular velocity $v$. However, the other cells, of which we take an average, are replaced by effective cells with mean coefficients computed in accordance with the formulae in Loucif (1981), generalized to both partial and complete frequency redistribution.

\section{Results and discussion}

To show the effect of the spatial correlation in the velocity field on the emergent profiles as well as the importance of fluctuations in the optical properties against the average, exhaustive calculations of emergent non-LTE profiles are performed. The calculations are made in the frame of systematic comparison between the two processes of redistribution in the three following cases: static medium, averaged turbulent medium, and a deviation from average case that corresponds to a particular realization of the turbulent medium. In the last case, the medium could be described as an averaged turbulent one correlated with a turbulent cell, the velocity of which deviates from the ensemble. The idea behind this particular model is to sketch astrophysical observational situations rather than to stick to the probabilistic description of the random process. With that assumption in mind, a parametric study is conducted over three key parameters: namely, the medium total opacity, the correlation length, and the velocity of the deviant turbulent cell.

\subsection{Static and averaged turbulent media case}

To give a general idea of the fundamental importance of the influence of the spatial correlation length in a turbulent medium (whatever the diffusion process assumed), Figs. 2 and 3 - where the total dimension $\tau$ of the medium is fixed, with an adequate cutting out of $\tau$ in $n=1,2, \ldots, \infty$ cells-allow us to have a great pattern from macro- $(n=1)$ to microturbulence $(n \rightarrow \infty)$ where mean emergent profiles are calculated, thus giving general direction to the behavior of both redistribution functions. In regard to emission lines, it is interesting to see at a glance in Fig. 2 a great similitude in behavior for both redistributions against the number $n$ of cells (or, inversely, against the correlation length $l=\tau / n)$, with the line core extending and the self-reversal at the line center getting deeper.

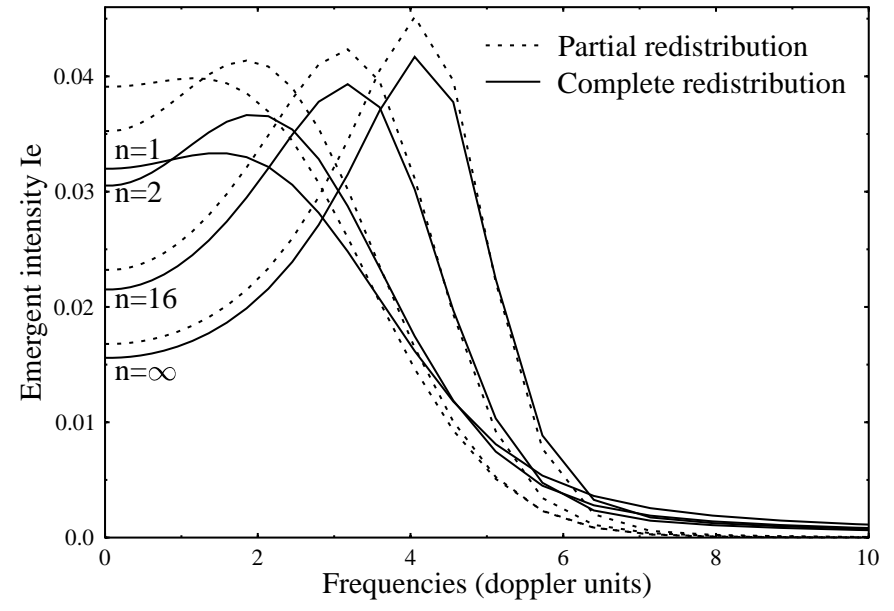

Fig. 2. The same example as in Magnan (1976) but with addition of Partial Redistribution (dashed lines) to compare to Complete Redistribution (solid lines), which presents an emergent intensity from a finite slab of optical thickness $\tau=320$ and a turbulence velocity $V_{\mathrm{T}}=2$ Doppler units. The medium is divided in $n$ equal layers. In order of decreasing central intensity, the number of effective layers is $n=1,2,16, \infty$.

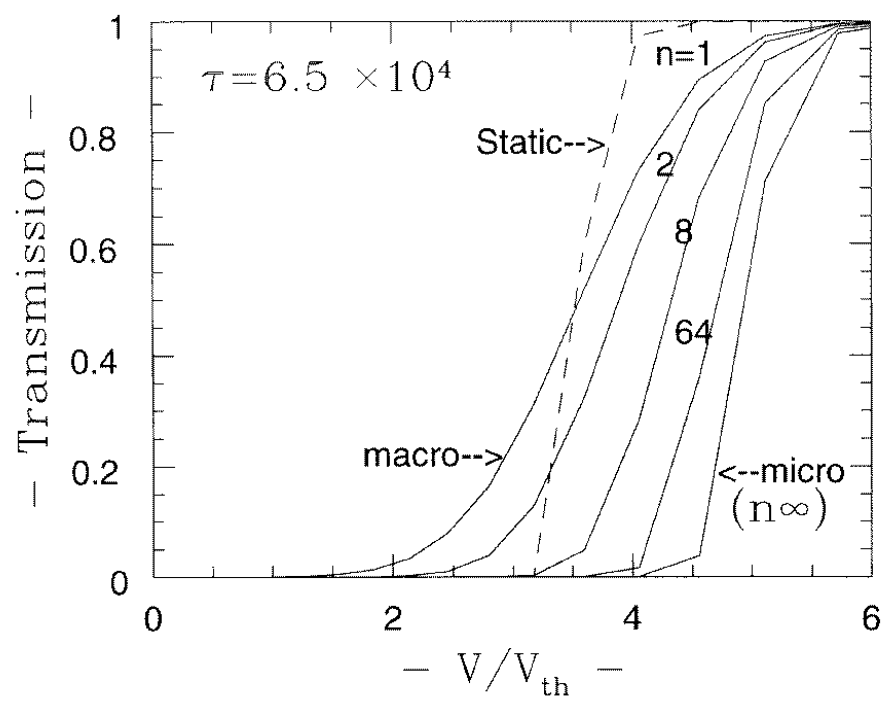

Fig. 3. The same as Fig. 2, but for the probability of transmission in the case of complete redistribution (after any number of scattering). $V$ and $V_{\text {th }}$ are respectively the velocity of cells and the thermal velocity. The static case is given for comparison.

This behavior, in fact, reflects that the amount of scattering is growing with the number of turbulent cells, reaching a saturation level when the microturbulence limit-a limit which corresponds to the thermal scale for scattering-is attained. The effective Doppler width is also increasing with the number of cells, which explains the larger and larger frequency at which the intensity reaches its maximum. This frequency also corresponds to the maximum escape probability of photons from the medium (Osterbrock 1962). Note, however, that in all cases shown, the intensity in the line core is always stronger for PRF than CFR, and is significantly larger for the macroturbulent limit than for the microturbulent limit. Also note that the far line wings 
Static medium
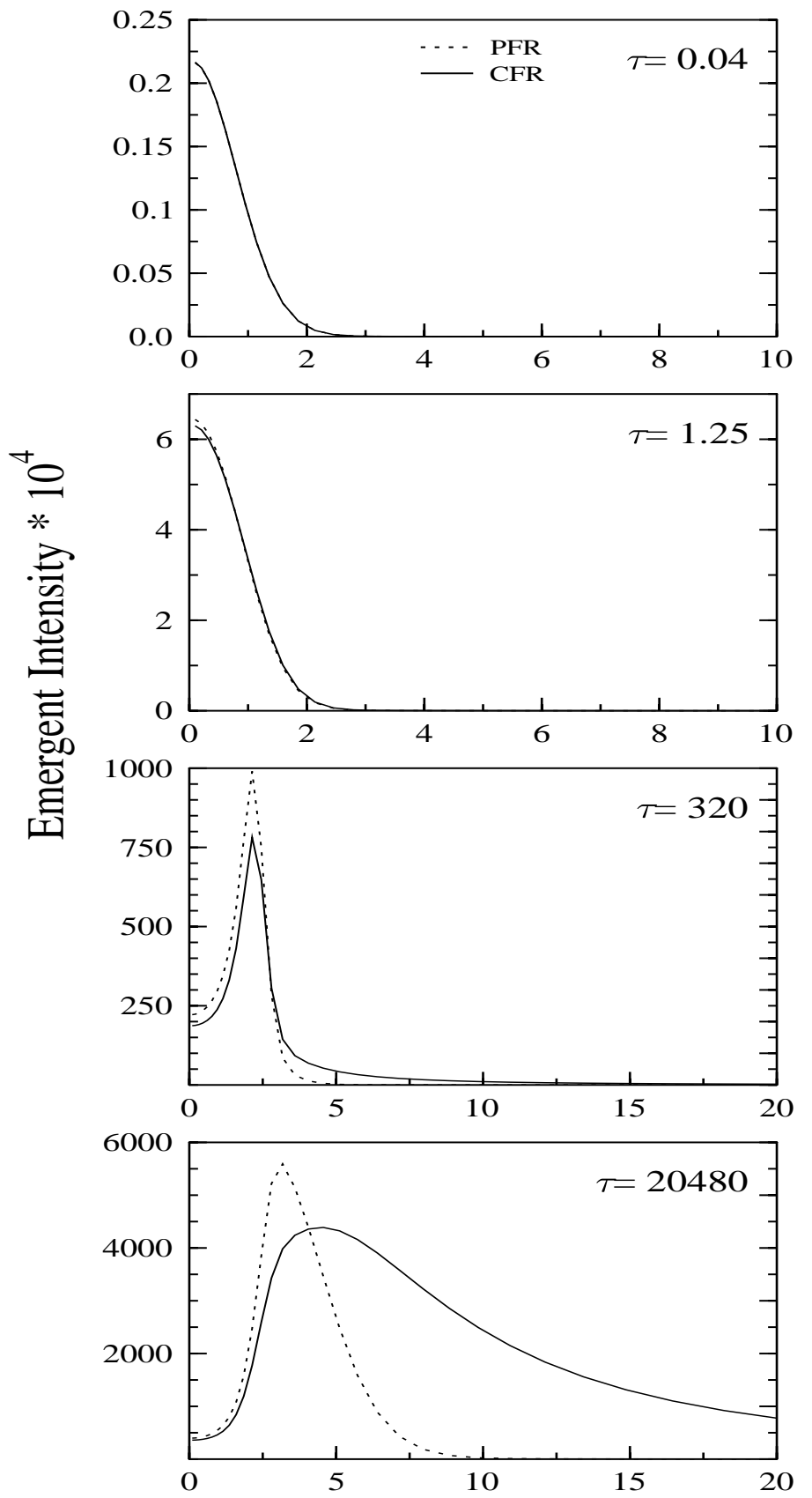

Averaged medium
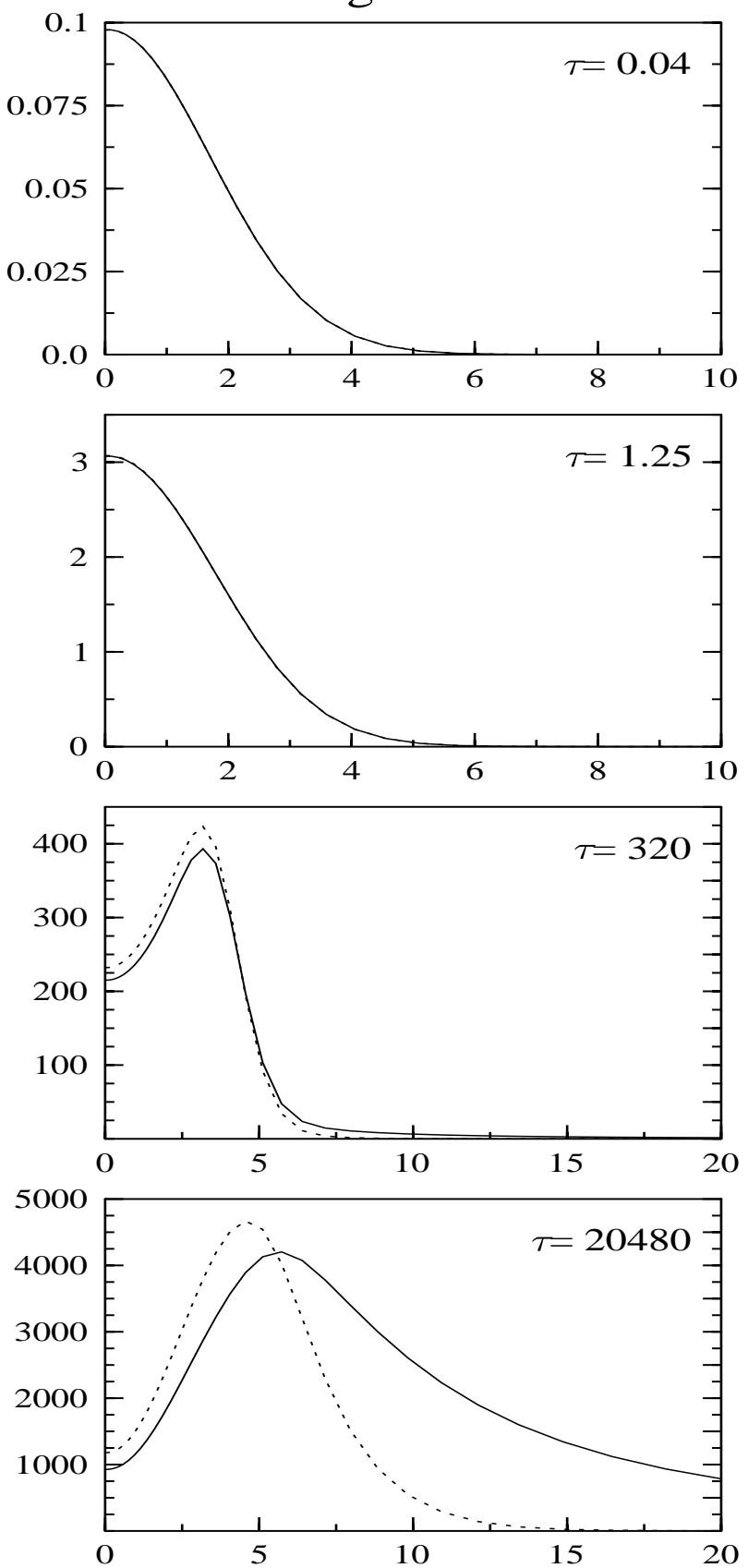

Frequencies (Doppler Units): v/a

Fig. 4. Example of half-profiles emerging from static (left column) and averaged turbulent medium $\left(V_{\mathrm{T}}=2\right.$, right column) for different values of $\tau$. Calculations were made for both partial (dashed lines) and complete (solid lines) frequency distribution. Note that for $\tau<1$, both distributions yield the same profile. Correlation length is $\frac{\tau}{16}$ (or $n=16$ ).

are expanding faster with CFR than with PFR when going from the macro- to the micro-limits. These properties are consequent to the intrinsic properties of the redistribution functions. Indeed, for PRF, Doppler redistribution only occurs in the line core, and for CFR, redistribution acts all over the line and is consequently more efficient in processing the photons' escape from the line core into the line wings. This explains why the line core intensity is weaker for CFR than for PFR. As the number of scattering relative to the number of cells is increasing, the line core saturates accordingly, making the intensity of the two functions comparable in the region while the wings are clearly much stronger and extended for CFR than for PFR. The PRF intensity is clearly much sharper at the frequency of maximum escape probability, a boundary where a transition in the frequency redistribution's process takes place for that function between the core and the wings of the line. 
For absorption lines, we add the static case in Fig. 3 in order to highlight the range of variation of the lines' width $(F W M H)$ against the correlation length. For the same total opacity, Fig. 3 shows that an observed line having a certain $F W M H$ could be interpreted erroneously in terms of temperature or abundance if the adequate correlation length is not used. This is of particular importance for light elements $(\mathrm{D}, \mathrm{H}, \mathrm{Li}$, etc.) abundance retrieval from absorption features in stellar and quasars spectra. Our results tend to confirm the general finding, also pointed out by recent publications in the literature, that the commonly used approximation of the microturbulent limit could underestimate the elements' abundance because of the important line broadening induced in that case (Hegmann \& Kegel 2000; Levshakov et al. 2001).

In the same line of reasoning, we also try to derive key differences and similitudes between partial and complete frequency redistribution as a function of the total opacity of the medium from the two commonly used applications for static and averaged turbulent media. Figure 4 shows a typical case generated for a range of opacities of the medium (with a correlation length of $\tau / 16$, or $n=16$, for the turbulent case). It is immediately apparent that no difference exists between the two redistributions for $\tau<1$ as far as both processes have the same absorption and emission profile - e.g., a Voigt function. As expected, for larger opacities, the classical differences between complete and partial redistribution are recovered, namely with a slightly deeper reversal and stronger wings for the CFR case (see above). It is interesting to note that these properties are conserved for a line formation in a turbulent medium, at least when quantities averaged over the turbulent velocity field are considered. As shown in Figs. 2-4, the differences between CFR and PFR hold for all correlation length scales and opacity regimes. Note, however, that a systematic line broadening is introduced by the turbulent velocity field by enhancing the probability that atoms scatter photons, an effect particularly efficient when the total opacity is large. As shown in Figs. 2 and 3 , this broadening is maximal in the microturbulence limit - namely, when the number of turbulent cells $n$ is much larger - thereby making the probability of scattering even greater.

\subsection{Departure from average turbulent media}

\subsubsection{Fluctuating medium: model description}

After considering respectively the correlation length and the total opacity effects in averaged turbulent media, in a third test we focus on the impact of a departure from average in a given realization of the velocity field. In this case, from our precedent hypothesis that photons have no memory of any but the last emitting cell's velocity, the turbulent medium is reduced to an average one, illuminating the last emitting cell that has a size equal to the spatial correlation length and moving with a particular velocity $v$ (e.g., Fig. 1). In probabilistic terms, we cannot in principle attach our picture to a realization of the random field. However, our assumption has the advantage of quite nicely sketching common astrophysical situations where distant or variable targets seem to be well-described by that picture of a large medium illuminating a "cell", the velocity of which "departs" from the ensemble. One could easily imagine the relative motion of a cloud against an ensemble of clouds along the line of sight, or think about protuberances in front of the solar disk as typical examples. So the picture we wish to study is much easier to depict as an observational case rather than in probabilistic terms related to the random field. Nonetheless, we tried to keep a relationship with the classical momenta of the field through the most probable velocity and the correlation scale length commonly used. A good illustration of our model is provided by recent HST observations of the Jovian H-Lyman- $\alpha$ emission line profile near the equatorial regions of the planet (Emerich et al. 1996). As shown in Fig. 5, the observed line profile evolves on a very short time scale. Each snapshot (having an exposure time of four minutes) of the emission line could be sketched by our simple picture of a large medium (the Jovian disk) illuminating one or more deviant cells, filling the field of view.

In the future, a self-consistent description will surely be needed to fully account for the in situ properties of the velocity field in distant objects with the related complex condition of remote observations - a description that is beyond the scope of this paper (e.g., Sedlmayr 1980; Magnan 1985).

\subsubsection{Fluctuating medium: results}

As expected, the resulting profiles are essentially sensitive to three factors: the total opacity of the medium, the correlation length of turbulence (corresponding to the optical depth of the individual cells), and the velocity of the last emitting cell. In order to separate effects and derive key properties of the impact of each of the three factors mentioned above, we first provide snapshots of the variation of the emission line profile in contrast respectively to the opacity (Fig. 6), the correlation length of the turbulence (Fig. 7), and finally, the velocity (Fig. 8). In a second step, for purposes of clarity, we group plots in such a montage that for each figure and a selected total opacity of the medium, three scale lengths are compared at the same time for several velocities of the moving cell relative to the average medium. In such a way, starting from Fig. 9 and continuing through Fig. 12, we scan the full spectrum of the turbulence correlation lengths from macro- to microturbulence from left to right, as well as a wide range of turbulent velocities from top to bottom.

All cases thus far generated suggest that both partial and complete redistribution give comparable general evolution; partial redistribution produces sharper spikes and much less broadening. The comparison of the top panels in Fig. 6 shows that no differences exist between CFR 

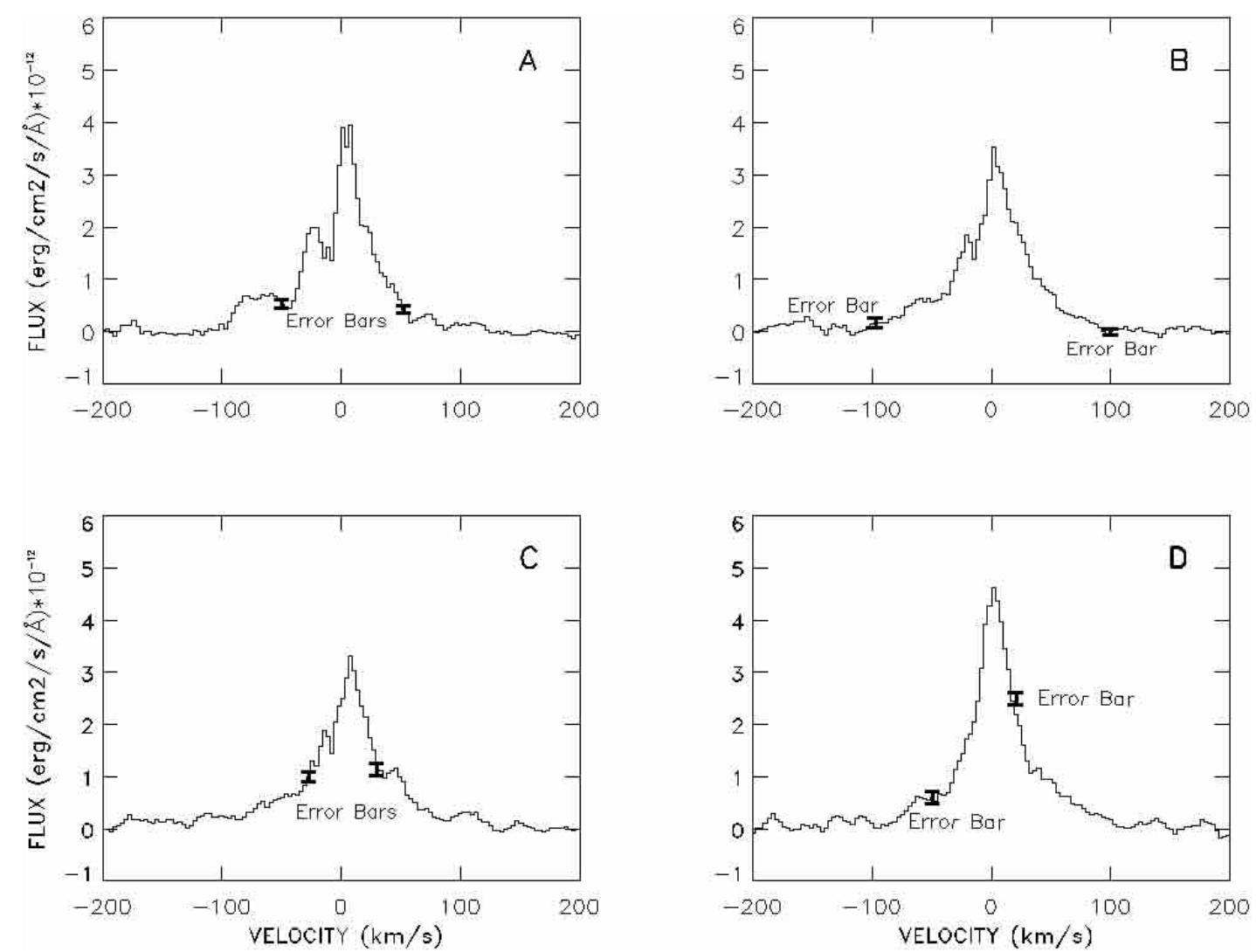

Fig. 5. Jupiter H-Lyman- $\alpha$ emission line profile obtained by HST/GHRS in 1995, as a time sequence running from panel A to D. For each snapshot, the exposure time lasted four minutes. Note the rapidly changing line shape from one panel to another. From Emerich et al. (1996).

and PFR for low opacities. For larger opacities, a clear difference appears between CFR and PFR for all correlation lengths and velocities (e.g., Figs. 7 and 8). It is interesting to note that the signature of the deviant cell is prominent when the correlation length is the largest i.e., close to the macroturbulence regime (Fig. 7). The effect of increased velocity tends to separate the components from the two media despite the correlation effect (Fig. 8). Intermediate values of the turbulence scale, commonly attached to the so-called mesoturbulence conditions, lead to the most complex line profiles. Based on the present work, line broadening is not the only signature of the presence of turbulence because asymmetries in the line profile could be obtained. The new signatures of the turbulent velocity field are obtained here in a unified description of the different regions contributing to the emitted line inasmuch as all parameters involved correspond to the genuine parameters that describe a turbulent medium: namely, the opacity, the correlation length of turbulence, and the velocity.

To understand the features so far obtained in the model line profiles, in the following we invoke coupling between Doppler effects consequent to the non-thermal velocity field and multiple scattering. Indeed, for an optically thick case, multi-scattering as well as thermal motion in a static medium usually leads to an emission line with a double peak corresponding to the maximum escape probability for photons (Osterbrock 1962; Adams 1973; Neufeld 1990). The introduction of turbulence induces respectively a specific and significant non-thermal broadening of the line and an additional emission component relevant to the last layer in front of the observer. Depending on the cell's opacity-which in our model corresponds to the optical size of the spatial correlation length-the individual line profile of the emitting layer may also show a double peak, but now it also overlaps on the profile from the averaged medium that illuminates it. We must keep in mind that only RT calculations, of the kind presented in this paper, account for the coupling between the different components of the medium in a unified picture. As a result, similar to conditions that prevail in media with a differential motion (Mihalas 1978), we are left with two/three/four peaks with irregular weights that may overlap, depending on the strength of velocity field invoked and the correlation length assumed. Also, as shown by Figs. 9-12, a line profile diagnosis based only on the number of components observed is not always reliable in view of the present results because of the difficulty in attaching each individual structure to the corresponding emitting region. For purposes of clarity, we generated several cases where we show opposite signatures in the emergent line profile that depends on the different parameters of importance: i.e., the correlation length and the velocity of the turbulent cell. The emergent line profiles are shown in the 


\section{Deviation from average \\ $(n=4)$}
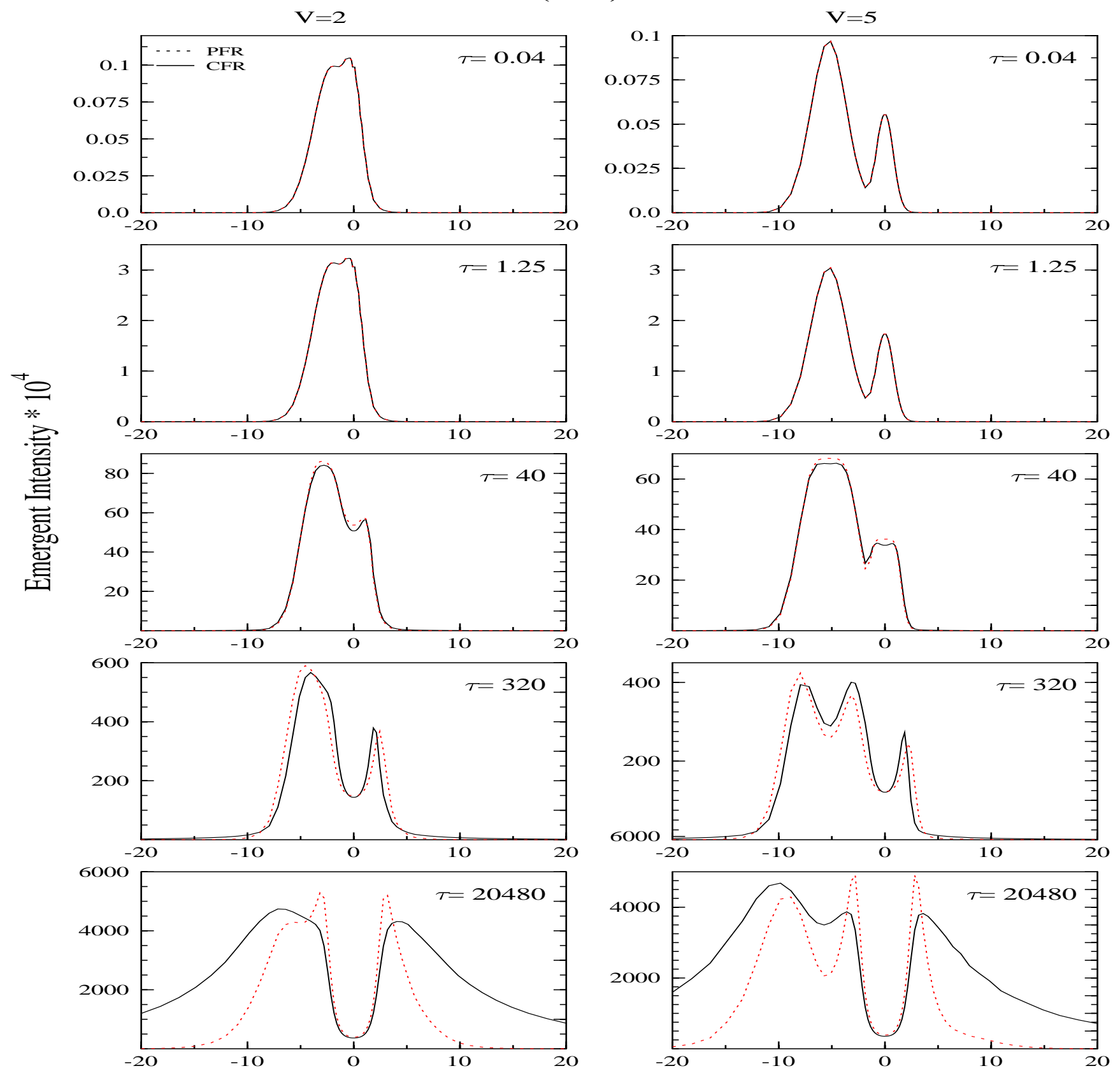

Frequencies (Doppler Units): v/a

Fig. 6. Example of individual profiles emerging from a turbulent medium for two particular velocities (2 and 5 , respectively) of the last emitting layer (surface layer). As in Fig. 4, the differences between the two frequency redistributions appear only for $\tau>1$. The correlation length is $\tau / 4$.

reference frame of the averaged large medium. In Fig. 7, where $n=4$ and $v=2$, the central self-reversal is centered on the velocity of the departing cell; compare this situation to a case in which $n=1024$ and $v=5$ whereby we may see the opposite signature, where the strongest self-reversal is now centered on the reference's frame rest frequency. This suggests that while spectra in the $n=1024$ case could be interpreted correctly by attaching each component of the spectrum to its corresponding emitting region, this is no longer possible for the $n=4$ case (Fig. 7) where a "plausible" interpretation would be to attach the strong self-reversal to the dominant medium and the weaker one to a "small region" that is moving toward the blue in the reference frame. As a logical extension, such reasoning would lead to the "discovery" that the target has an intrinsic motion not corrected-for at that stage of the data analysis. The interpretation could be even more complicated by the natural instrumental psf effect and the 


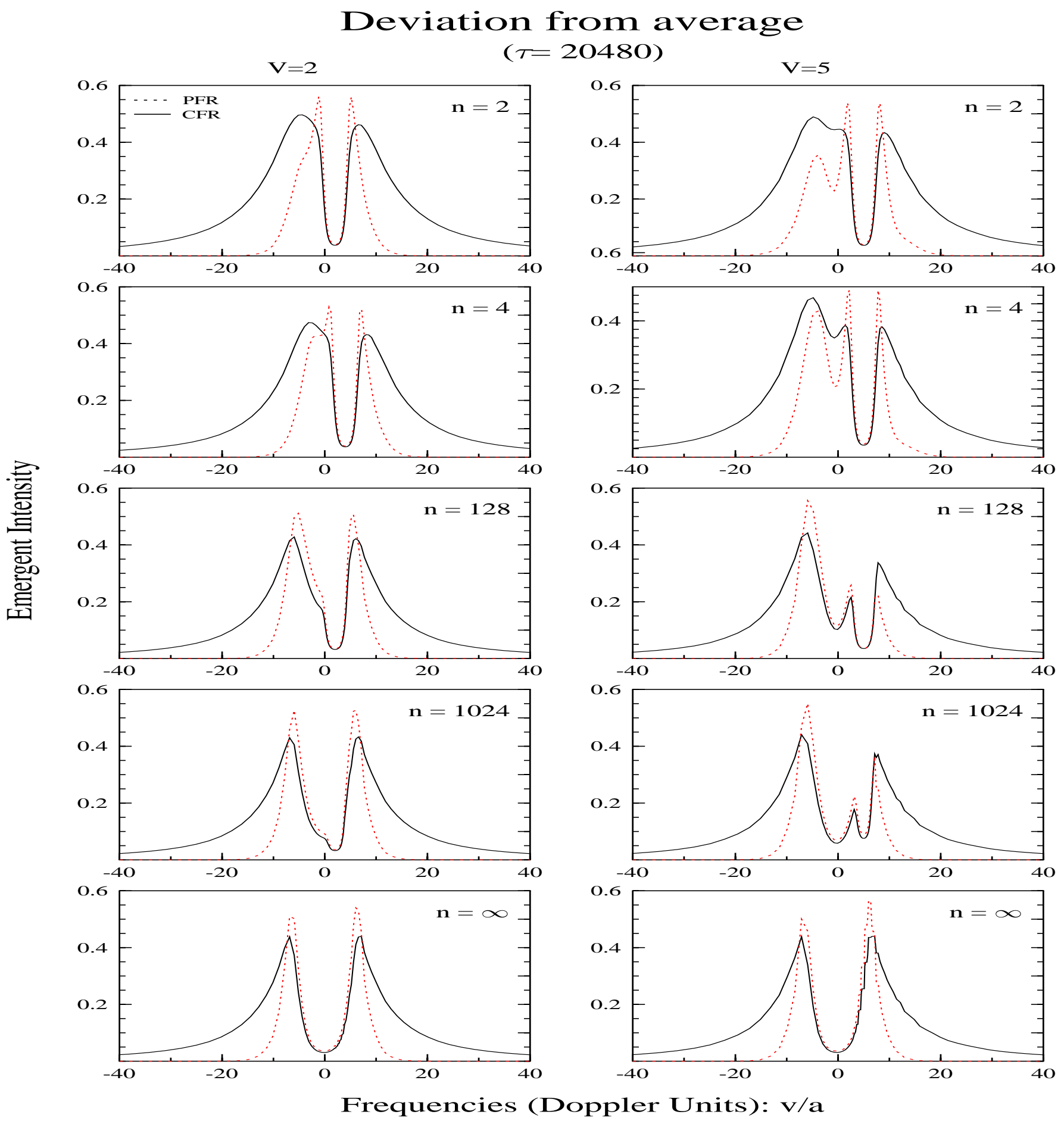

Fig. 7. The same as in Fig. 6, but focusing on the model's sensitivity to the correlation length for a fixed total opacity of $\tau=20480$ of the medium. The correlation length is $l=\frac{\tau}{n}$.

detectors' noise that may hide some spectral features. The confusion introduced by the interplay between the correlation length and the turbulent velocity, as illustrated by several panels in Figs. 8-12, therefore makes a direct interpretation of multi-component spectra observed toward some stellar objects difficult, particularly if the spectral range is rich in emission lines. The unified description that we propose to sketch of a turbulent medium may help to overcome these difficulties.
For astrophysical objects close enough to Earth to be observed with different phase angles, or extended enough in the sky to measure the center-to-limb variation of emission lines (Sun, planets, etc.), our model calculations shown in Fig. 6 reveal a strong dependency of the line shape on the medium's opacity. This property may be used to help build a diagnostic tool to discriminate between the two redistribution functions by scanning the same region at different locations on the disk and thus 


\section{Deviation from average $(\tau=327680, \mathbf{n}=8192)$}
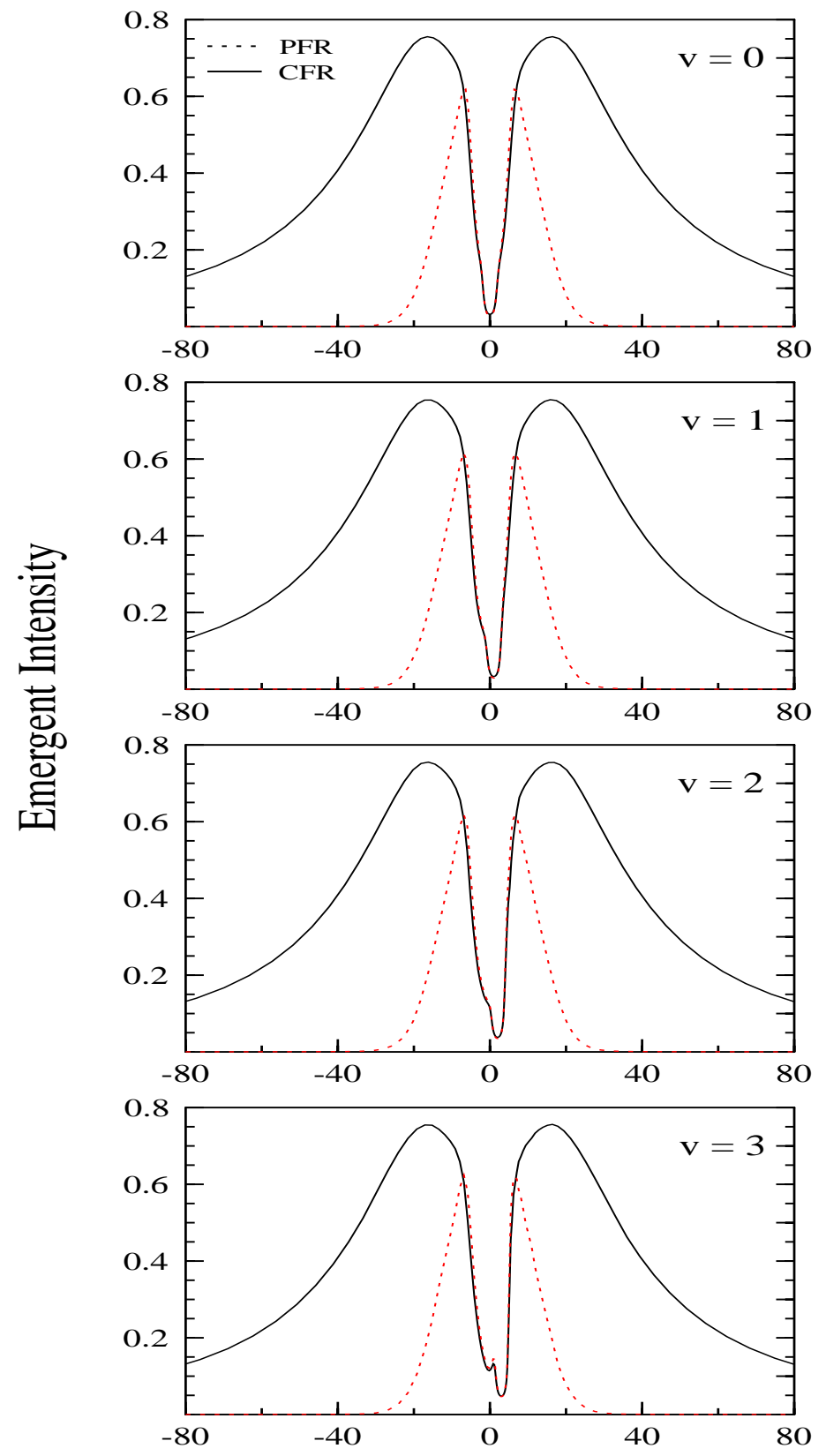
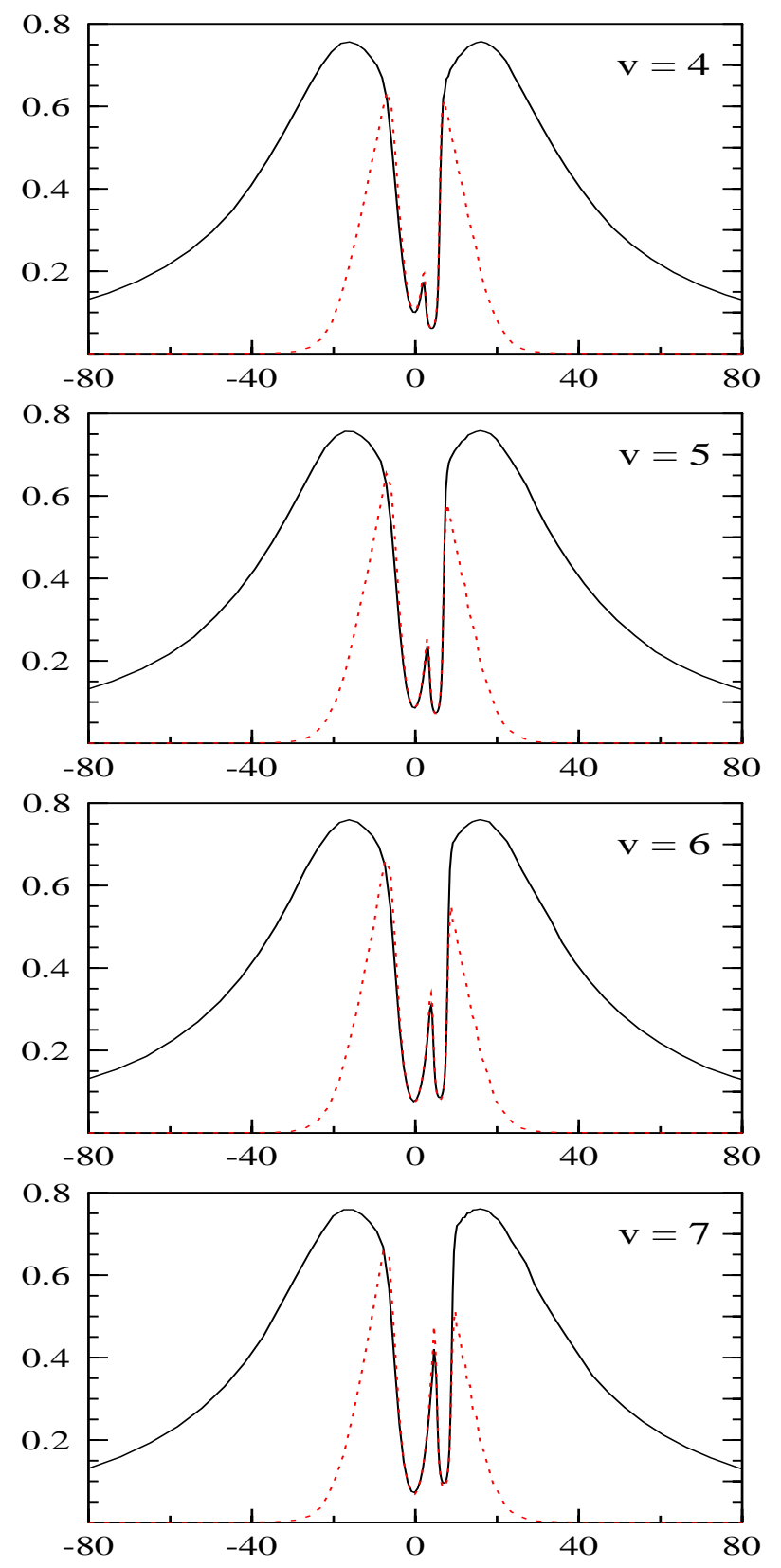

Frequencies (Doppler Units): v/a

Fig. 8. The same as in Figs. 6 and 7, but against the velocity of the "departing" cell for a fixed opacity of the medium $\tau=327680$ and a correlation length of $\tau / 8192$.

probing different opacity regimes. This technique was successfully applied to interpret the Jovian equatorial H-Lyman- $\alpha$ emission using line profiles observed by IUE respectively from the center and limb of the planetary disk (Ben-Jaffel et al. 1993). Because of limitations inherent to the data quality, invoking thermal and microturbulence broadening helped to reproduce the observed line profiles (Ben-Jaffel et al. 1993). However, more recent observations obtained by HST of the same Jovian equatorial region show high-resolution Lyman- $\alpha$ emission line profiles to be very disturbed on a time scale of a few minutes (Emerich et al. 1996). As shown in Fig. 13, classical RT models based on thermal or microturbulence broadening could not reproduce the observed line shape. The present non-LTE model of radiation transfer in its "departure from average" version will be applied in the near future in order to interpret the HST data. 


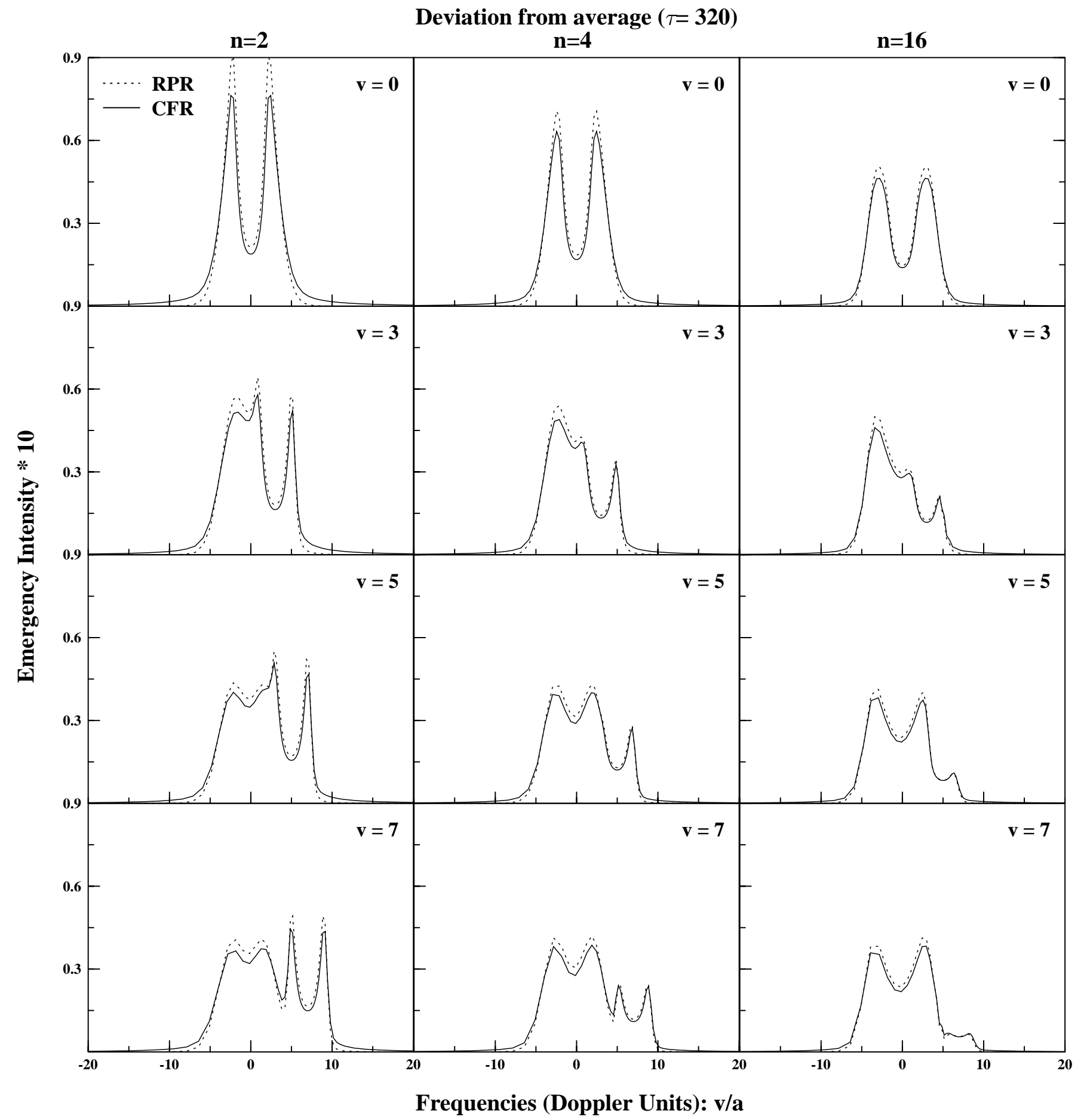

Fig. 9. Emergent emission profile from a turbulent medium with a "departing" cell described by our unified model as a function of the turbulent velocity (top to bottom). Effect of the correlation length for total opacity of the medium $\tau=320$ (left to right). At $n=16$ and beyond, the microturbulence limit is attained and line profiles remain unchanged. CFR (solid line) and PFR (dashed line).

\section{Conclusions}

We computed line profiles escaping from a plane parallel slab intended to mimic conditions that prevail in turbulent media with a finite correlation length of the velocity field, including multiple scattering effects through complete or partial frequency redistribution functions.
The problem is in non-LTE conditions, and so we followed Loucif \& Magnan (1982) in order to describe the line formation in respectively a static medium, an averaged turbulent medium, and the so-called deviation from average medium. The results so far obtained reveal that the velocity field is indeed crucial for determining the line shape. As expected, there is almost no difference between CFR 


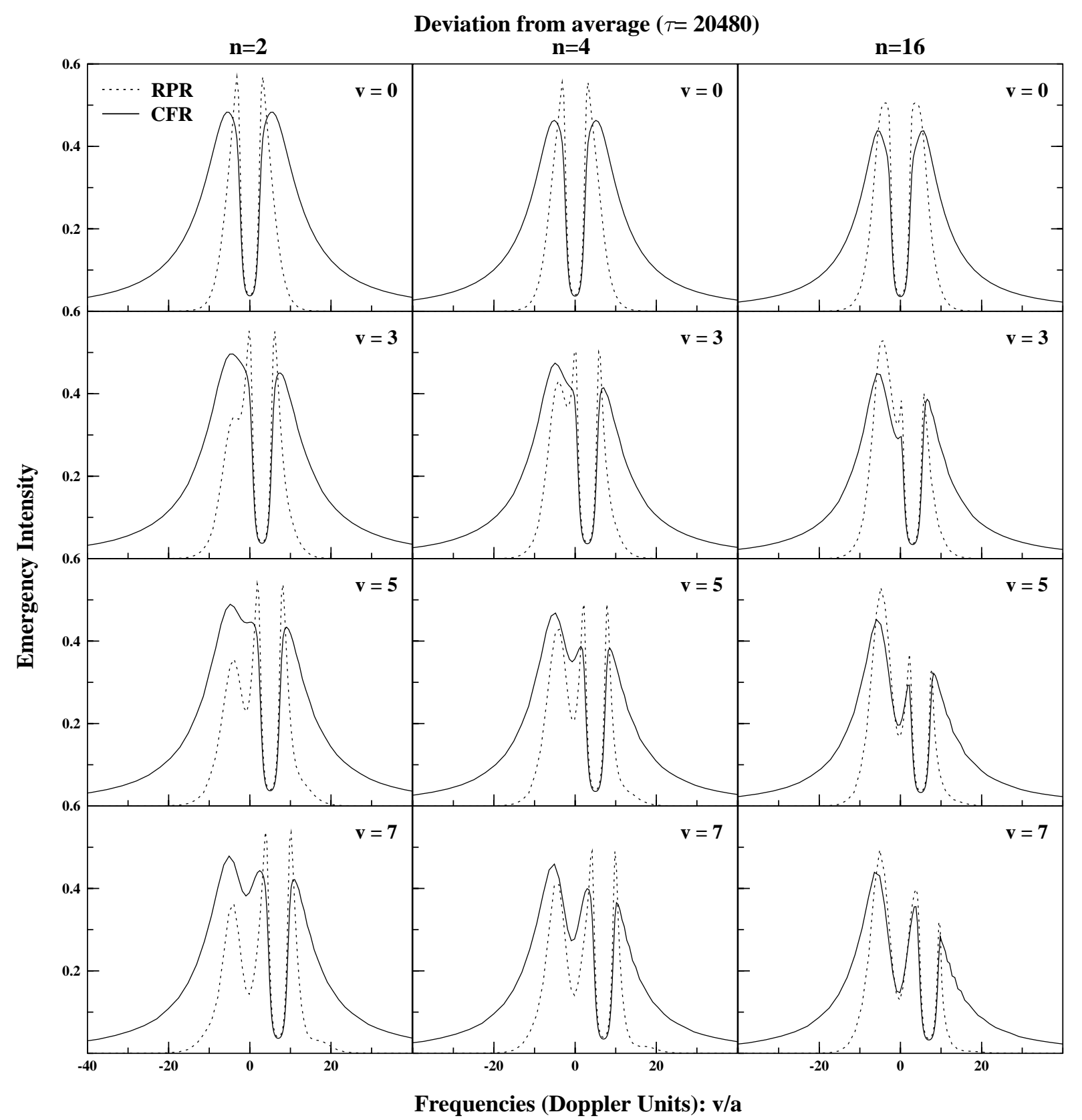

Fig. 10. Same as in Fig. 8 but with the total opacity of the medium as $\tau=20480$ and three correlation lengths: $\tau / 2, \tau / 4$, and $\tau / 16$.

and PFR functions for any small opacity of the medium. For larger opacities, the emergent spectra show a significant difference between CFR and PFR, with intensity increasing in the line core for PFR, and in the far wings for CFR. Also, in the presence of a turbulent velocity field, the line broadening obtained with CFR is much larger than with PFR. However, the line structures are somewhat smoothed out when compared to the static case (e.g., Fig. 6). When considering deviation from average conditions, as shown in Figs. 6-12, the velocity "fluctuation" produces extra components that might, in some cases, hide the main line, thereby making the spectral diagnosis very difficult. Indeed, there is a kind of interplay respectively between the turbulence correlation length, medium opacity, and deviant cell's velocity that produces spectra which do not lend themselves to straightforward interpretations if the real conditions that prevail in the medium are not known. Depending on the medium's opacity, we showed that the emergent line profiles are mainly controlled by the finite size of correlation length and the fluctuation of the turbulent medium. The strength of the radiative transfer model proposed in this study is that it provides a unique picture to describe the turbulent medium as a whole, while the emergent lines could have multiple spectral components.

As shown by recent high-resolution observations (SOHO, HST, FUSE, etc.) of different astrophysical sites, and confirmed by our turbulence model with its 


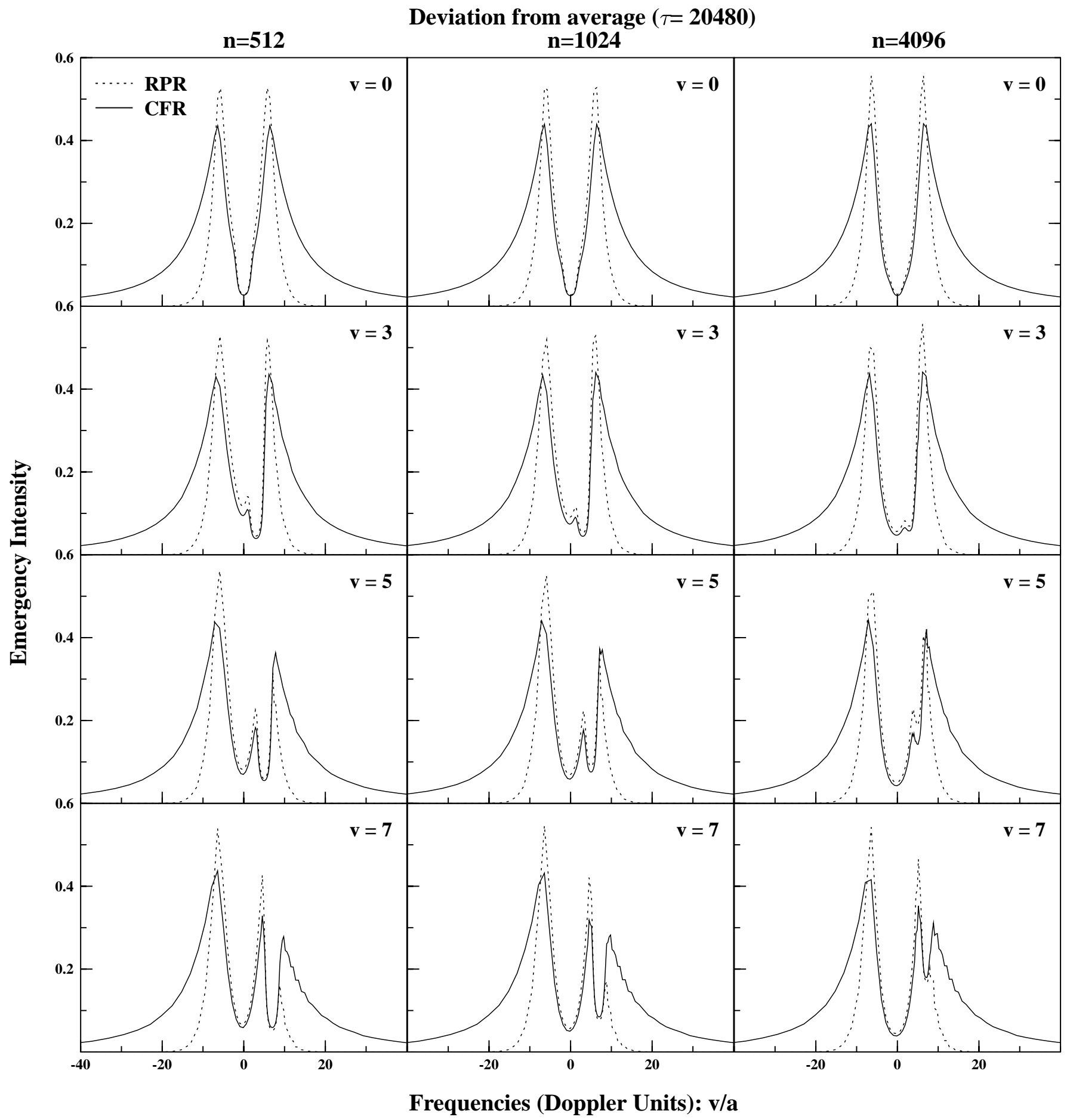

Fig. 11. Same as in Fig. 9 but with three more correlation lengths: $\tau / 512, \tau / 1024$, and $\tau / 4096$. The three additional cases are shown in order to trace the emergent line profile evolution and contrast it to the correlation length until the microturbulence limit is attained (around $\tau / 4096$ ).

"departure from average" version, the very notion of a kind of "mean" atmosphere could be in fact meaningless. This is crucial for the interpretation of data arising from rich wavelength windows where dense molecular lines overlap. This is also of particular importance for absorption lines that are observed in stellar and quasars spectra typically used to derive interstellar or galactic abundances. The very possibility of the occurrence of a velocity fluctuation may corrupt the abundance measure and entail heavy consequences for the physical interpretation $(\mathrm{D} / \mathrm{H}, \ldots)$. This particular aspect will be treated in a forthcoming paper.

Acknowledgements. The authors acknowledge support from the Centre National de la Recherche Scientifique (CNRS) and the Institut National des Sciences de l'Univers (INSU) of France under the programs PNST and PNP. LBJ acknowledges support from CNES under the project INSPIRE. 


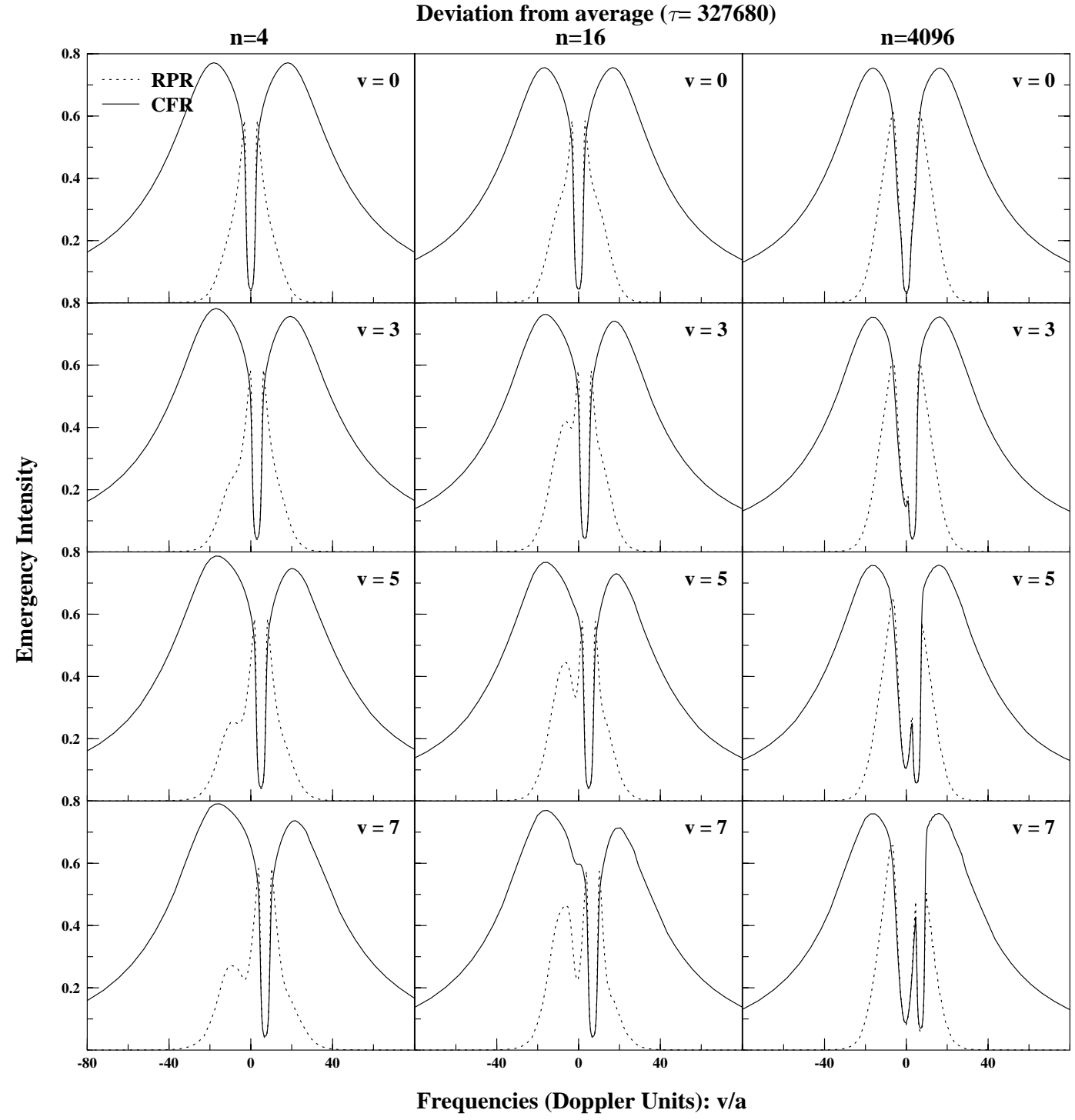

Fig. 12. Same as in Fig. 8 but with a total opacity of the medium as $\tau=327680$ and three correlation lengths: $\tau / 4$, $\tau / 16$, and $\tau / 4096$.

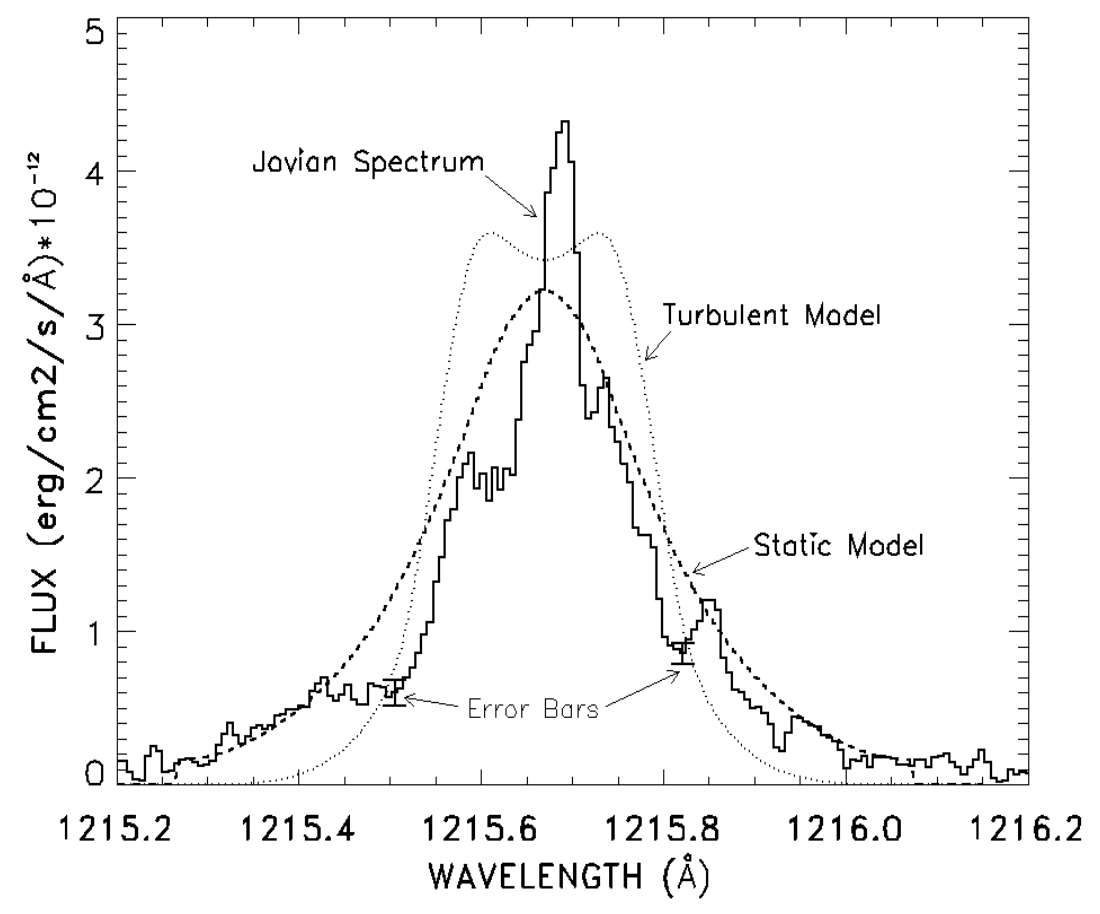

Fig. 13. Jovian H-Lyman $\alpha$ emission line profile as observed by HST/GHRS near the equatorial region of the planet. A static model and a turbulence model, including microturbulence broadening, are also shown. Neither model could recover the structure of the observed line profile (from Emerich et al. 1996). 


\section{References}

Adams, T. F. 1972, ApJ, 174, 439

Albrech, M. A., \& Kegel, W. H. 1987, A\&A, 176, 317

Ben Jaffel, L., Clarke, J. T., Prange, R., et al. 1993, Geophys. Res. Lett., 20, 747

Ding, M. D., \& Schleider, H. 1998, A\&A, 332, 767

Emerich, C., Ben Jaffel, L., Clarke, J. T., et al. 1996, Science, 273,1085

Gontikakis, C., Vial, J.-C., \& Gouttebroze, P. 1997, A\&A, 325, 803

Hegmann, M., \& Kegel, W. H. 2000, A\&A, 359, 405

Hummer, D. G. 1962, MNRAS, 125, 21

Levshakov, S. A., Kegel, W. H., \& Takahara, F. 1999, MNRAS, 302, 707

Levshakov, S. A., Kegel, W. H., \& Agofonova, I. 2001, A\&A, 373,836

Loucif, M. L. 1981, Thèse de troisième cycle, Université Paris 7
Loucif, M. L., \& Magnan, C. 1982, A\&A, 112, 287

Magnan, C. 1976, J. Quant. Spec. Radiat. Transf., 16, 281

Magnan, C. 1985, A\&A, 144, 186

Mihalas, D. 1978, Stellar Atmospheres, 2nd ed. (Freeman, San Francisco)

Neufeld, D. A. 1990, ApJ, 350, 216

Osterbrock, D. E. 1962, ApJ, 135, 195

Piehler, G., \& Kegel, W. H. 1995, A\&A, 297, 841

Sedlmayr, E. 1980, in Turbulence, IAU Coll. 51, London, Canada; Lectures Notes in Physics, 114 (Springer, Berlin, Heidelberg, New York), 195

Sobolev, V. V. 1963, A treatise on radiative Transfer (Translation S.O. Gaposchkin) (van Nostrand, New York)

Vial, J.-C., Gouttebroze, P., Artzner, G., \& Lemaire, P. 1979, Sol. Phys., 61, 39

Warren, H. P., Mariska, J. T., \& Wilhem, K. 1998, ApJS, 119, 105 